\title{
Self-consistent evaluation of quark masses in three flavor crystalline color superconductivity
}

\author{
N. D. Ippolito, G. Nardulli and M. Ruggieri \\ Dipartimento di Fisica, Università di Bari, I-70126 Bari, Italia \\ and \\ I.N.F.N., Sezione di Bari, I-70126 Bari, Italia \\ E-mail: nicola.ippolito@ba.infn.it, giuseppe.nardulli@ba.infn.it, \\ marco.ruggieri@ba.infn.it
}

\begin{abstract}
We present a self-consistent evaluation of the constituent quark masses in the three flavor Larkin-Ovchinnikov-Fulde-Ferrell (LOFF) phases of QCD, employing a Nambu-Jona Lasinio model. This result allows to determine the window for values of the baryonic chemical potential where the LOFF state is energetically favored.
\end{abstract}

KeYwords: High density QCD, Nambu-Jona Lasinio model, Crystalline color superconductivity, Constituent quark masses. 


\section{Contents}

1. Introduction 1

2. Nambu-Jona Lasinio model at $\mu=0$

3. Constituent masses in the LOFF phases 5

4. Conclusions 11

\section{Introduction}

The phase diagram of three flavor QCD in the low temperature and high density regime has recently received considerable theoretical attention. Quark matter in these conditions is expected to be a color superconductor [1, 2, 3] and this state of hadronic matter definitely deserves a careful study. On the other hand the core of the compact stars is the place where this exotic state of matter might be found. Therefore the study of color superconductivity is interesting not only theoretically, for it allows a deeper knowledge of the phase diagram of QCD, but also phenomenologically, since this phenomenon might be revealed in compact stellar objects.

At very high densities the color superconductor is expected to be in the color-flavor locked state (CFL) [3], characterized by a spin zero homogeneous condensate with nine gapped fermion excitations. When density is decreased, the electrical and color neutrality conditions together with finite quark mass effects produce a mismatch of the Fermi surfaces of the pairing fermions. In this case the CFL state has to be replaced by some less symmetric phase. Among them the gapless CFL (gCFL) [4, the gapless 2SC (g2SC) [5] and the $S=1$ color superconductors [6] have been extensively studied.

Besides the aforementioned color superconductive phases one can also consider inhomogeneous states, produced when the fermion pair has nonvanishing total momentum. This possibility was first discussed in a condensed matter physics context [0]; the corresponding phase is known as the LOFF state. In QCD the LOFF color superconductive phase has been studied firstly in the two flavor case [8] and then extended to the more interesting three flavor case [9, 10, 11, 12]. LOFF states are interesting since allow for pairing in conditions of highly mismatched Fermi surfaces. Moreover it has been shown that they do not suffer of the chromo-magnetic instability problem [13, 14, 15, 16, 17], contrarily to the homogeneous gapless phases [18].

In the last years the studies on color superconductivity have been improved by a selfconsistent treatment of the constituent quark masses in homogeneous phases [19, 20, 21, [22, 23]. These studies are based on phenomenological models, because their aim is to 
study quark matter in an intermediate density regime where the use of perturbative QCD is questionable. The favorite approach is based on the Nambu-Jona Lasinio (NJL) model 24] (see 25, 26, 27] for reviews). This model embodies the symmetries of QCD. Although it suffers many limitations, mainly due to the lacking of gluons as dynamical fields, it is believed to capture some basic physics of the strong interactions and to describe the qualitative features of the QCD phase diagram.

The aim of this paper is to evaluate in a self-consistent way the constituent quark masses in the three flavor LOFF phase of QCD. Our approach is similar to that employed in Refs. 21, 22, 23] for the homogeneous phases and therefore represents an extension of these works to the LOFF color superconductive phases. To test the robustness of our results we will consider two ways to treat the ultraviolet cutoff at $\mu \neq 0$. In the first case we will use, as usual in the literature, the same cutoff employed at $\mu=0$; in the second case we treat the cutoff by the method discussed in [28], where a link between the cutoff and the coupling constants is assumed. The two methods provide rather similar results and in both cases windows of values of the chemical potential are found where the LOFF phase is energetically favored.

The plan of the paper is as follows. In Section 20 we review the NJL model at $\mu=0$ and in particular we extend some of the results that were obtained in [28] for massless quarks to the case of massive quarks. In Section 3 we extend these results at $\mu \neq 0$. We minimize the free energy for the order parameters in the $\bar{q} q$ and $q q$ channels. For the $q q$ condensate we consider two different LOFF states: the one plane wave ansatz and two structures based on cubic symmetry, one having 8 plane waves and another having 16 plane waves. These two cases were considered in Refs. [9, 10] and [11] respectively, assuming zero masses for the up and down quarks and taking the strange quark mass $M_{s}$ as a free parameter. The result of these papers is that the LOFF phase is favored for certain values of the parameters $M_{s}^{2} / \mu$. Since now we compute $M_{s}$ as a function of $\mu$ we are able to determine a range of values of $\mu$ where the LOFF phase is energetically favored. Finally in Section 1 we draw our conclusions.

\section{Nambu-Jona Lasinio model at $\mu=0$}

In this Section we review the NJL model at zero quark chemical potential. We consider a system of $u, d$ and $s$ quarks described by the lagrangian

$$
\mathcal{L}=\sum_{f} \bar{\psi}_{f} i \partial_{\mu} \gamma^{\mu} \psi_{f}+\mathcal{L}_{\text {mass }}+\mathcal{L}_{4}+\mathcal{L}_{6}
$$

where the sum is over the flavors $f(=1,2,3$ for $u, d, s)$. The mass term in the lagrangian is

$$
\mathcal{L}_{\text {mass }}=-\sum_{f} m_{f} \bar{\psi}_{f} \psi_{f}
$$


and $m_{f}$ is the current mass. In this paper we shall assume from the very beginning $m_{u}=m_{d}$. The NJL four-fermion and six-fermion interaction Lagrangians are 25, 26]

$$
\begin{aligned}
& \mathcal{L}_{4}=G \sum_{a=0}^{8}\left[\left(\bar{\psi} \lambda_{a} \psi\right)^{2}+\left(i \bar{\psi} \gamma_{5} \lambda_{a} \psi\right)^{2}\right], \\
& \mathcal{L}_{6}=-K\left[\operatorname{det} \bar{\psi}_{f}\left(1+\gamma_{5}\right) \psi_{f^{\prime}}+\operatorname{det} \bar{\psi}_{f}\left(1-\gamma_{5}\right) \psi_{f^{\prime}}\right],
\end{aligned}
$$

where $\lambda_{a}$ are the Gell-Mann matrices in flavor space $\left(\lambda_{0}=\sqrt{2 / 3} \mathbf{1}_{f}\right)$ and the determinant is in flavor space as well. $\mathcal{L}_{4}$ and $\mathcal{L}_{6}$ describe two-body and three-body interactions respectively. In the Hartree approximation one has for $\mathcal{L}_{4}$ the result

$$
\mathcal{L}_{4}=4 G \sum_{f} \sigma_{f} \bar{\psi}_{f} \psi_{f}-2 G \sum_{f} \sigma_{f}^{2}
$$

and for $\mathcal{L}_{6}$

$$
\mathcal{L}_{6}=-2 K \sum_{f} \sigma_{f+1} \sigma_{f+2} \bar{\psi}_{f} \psi_{f}+4 K \sigma_{u} \sigma_{d} \sigma_{s}
$$

In deriving these equations one assumes condensation only in the quark-antiquark channel and treats consistently the number of colors $N_{c}$ assuming that $K N_{c}^{2} \sim O(1)$, neglecting $1 / N_{c}$ corrections 25, 26]. For each flavor $f$ one has

$$
\sigma_{f}=-i N_{c} \operatorname{tr} S_{f}
$$

where $S_{f}$ is the propagator of the quark of flavour $f, N_{c}$ is the number of colors, and the trace is on spinor indices only. By definition $\sigma_{4}=\sigma_{u}, \sigma_{5}=\sigma_{d}$.

The six-fermion t'Hooft term mixes flavors and originates from the $U_{A}(1)$ breaking contribution. At zero density it has the effect to lift the degeneracy of the $\eta$ and $\eta$ ' mesons. Note that in a different approximation scheme it also modifies the four-fermion giving rise to an effective four-fermion coupling constant. Using (2.5) and (2.6) the self-consistent equations for the constituent quark masses at zero baryon density read [25, 22]

$$
M_{f}=m_{f}-4 G \sigma_{f}+2 K \sigma_{f+1} \sigma_{f+2},
$$

where

$$
\sigma_{f}=-\frac{3 M_{f}}{\pi^{2}} \int_{0}^{\Lambda} \frac{p^{2}}{\sqrt{p^{2}+M_{f}^{2}}} d p .
$$

To fix the parameters of the model we use as an input the pion decay constant $f_{\pi}=93$ $\mathrm{MeV}$, the pion and kaon masses $m_{\pi}=135 \mathrm{MeV}$ and $m_{K}=497 \mathrm{MeV}$, the ratio

$$
\frac{<\bar{s} s>_{0}}{<\bar{u} u>_{0}} \simeq 0.80
$$

of the strange to the up chiral condensate, and the values of the current quark masses. In detail the equation for $f_{\pi}$ is

$$
f_{\pi}^{2}=-4 i N_{c} M_{u}^{2} I\left(0, M_{u}, M_{u}\right)
$$


where

$$
I\left(k^{2}, M_{1}, M_{2}\right)=\int \frac{d^{4} p}{(2 \pi)^{4}} \frac{1}{\left[(p+k / 2)^{2}-M_{1}^{2}\right]\left[(p-k / 2)^{2}-M_{2}^{2}\right]},
$$

From this relation we get $M_{u}(\Lambda)$. We note that the quark condensates are given by [26]

$$
\left\langle\bar{\psi}_{f} \psi_{f}\right\rangle_{0}=\sigma_{f}+\frac{3 m_{f}}{\pi^{2}} \int_{0}^{\Lambda} \frac{p^{2}}{\sqrt{p^{2}+m_{f}^{2}}} d p
$$

Using $m_{u}=m_{d}=5.5 \mathrm{MeV}$, and $M_{u}(\Lambda)$ we get $\left\langle\bar{\psi}_{f} \psi_{f}\right\rangle_{0}$ as a function of the cutoff $\Lambda$. To obtain $M_{s}(\Lambda)$ we use Eq. (2.13) evaluated for $f=3$ and Eq. (2.10). For the current strange mass we take $m_{s}=135.4 \mathrm{MeV}$, which is in the range of values allowed by QCD sum rules [29] and other methods [30] for the running current mass at the subtraction point of $1 \mathrm{GeV}$ and is to be preferred in view of a fit to the $\eta$ and $\eta^{\prime}$ mass, see below. The two constituent masses are reported in Fig. 1 as a function of the cutoff $\Lambda$.

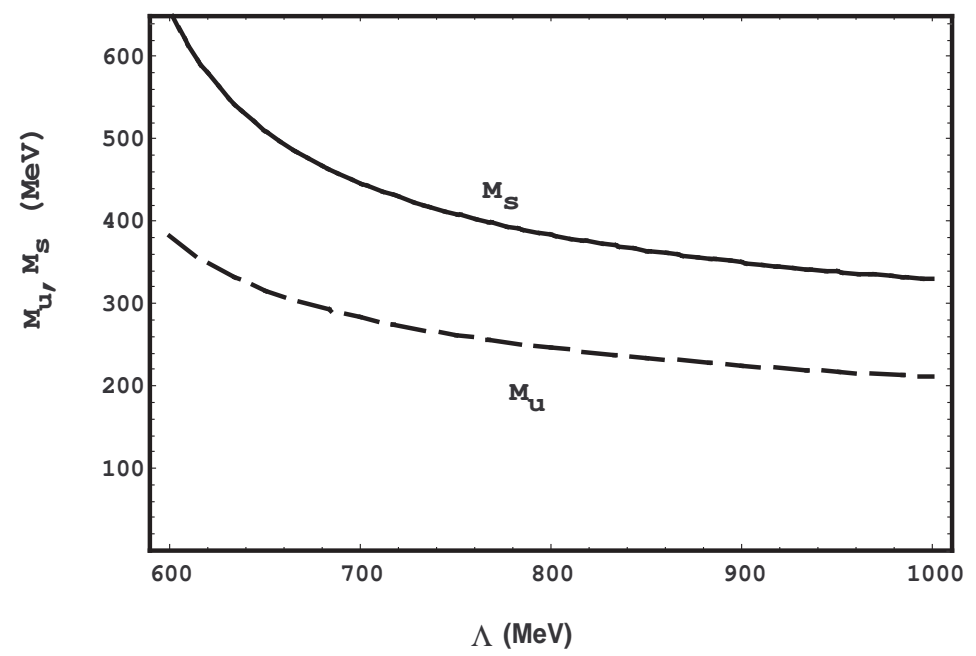

Figure 1: Constituent quark masses $M_{u}$ and $M_{s}$ as a function of the cutoff $\Lambda$.

The equations giving the neutral pion and the neutral kaon masses represent the massshell condition and are obtained in the random phase approximation [25]. They are as follows:

$$
\begin{aligned}
& 1-\left(2 G-K \sigma_{s}\right) \Pi\left(m_{\pi^{0}}^{2}, M_{u}, M_{u}\right)=0, \\
& 1-\left(2 G-K \sigma_{u}\right) \Pi\left(m_{K}^{2}, M_{u}, M_{s}\right)=0,
\end{aligned}
$$

with

$$
\Pi\left(k^{2}, M_{1}, M_{2}\right)=-6 i \int \frac{d^{4} p}{(2 \pi)^{4}} \frac{k^{2}-4 p^{2}+4 M_{1} M_{2}}{\left[(p+k / 2)^{2}-M_{1}^{2}\right]\left[(p-k / 2)^{2}-M_{2}^{2}\right]},
$$

and allow to get $G$ and $K$ as a function of $\Lambda$. These results are in Fig. 2 .

Finally we fix the value of the cutoff by requiring that the gap equation Eq. (2.8) be satisfied for the strange quark: on the l.h.s of Eq. (2.8) we use the $M_{s}(\Lambda)$ shown in Fig. 1; 


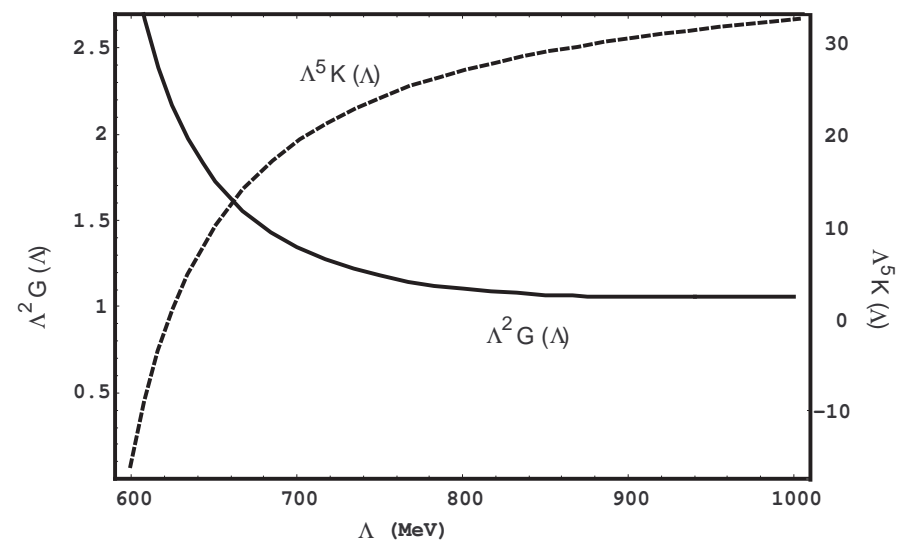

Figure 2: Couplings $\Lambda^{2} G(\Lambda)$ and $\Lambda^{5} K(\Lambda)$ as a function of $\Lambda$.

the same function is used on the r.h.s. to evaluate $\sigma_{s}(\Lambda)$, and $G$ and $K$ are replaced by the functions $G(\Lambda), K(\Lambda)$ plotted in Fig. 2. If we denote by $\Lambda_{0}$ such a value of the cutoff, valid at zero density and zero temperature, we get

$$
\Lambda_{0} \simeq 643 \mathrm{MeV} .
$$

We note that by this method one has another solution, with a smaller value of $\Lambda_{0}$. However using equations analogous to (2.14), (2.15) for the $\eta$ and $\eta^{\prime}$ mesons (for the relevant formulae see Section IV B of Ref. [25]) and the value 2.17) one gets as a result $m_{\eta}=481 \mathrm{MeV}$ (exp. $548 \mathrm{MeV})$ and $m_{\eta^{\prime}}=924 \mathrm{MeV}(\exp .956 \mathrm{MeV}$ ), while the use of the smaller cutoff would produce a worse fit to the experimental masses. As a check of the procedure we compute also $f_{K}$ [25]. We get $f_{K} \simeq 98 \mathrm{MeV}$ (exp. $112 \mathrm{MeV}$ ). The values of the other parameters, computed at $\Lambda_{0}$, are $M_{u}=M_{d}=324 \mathrm{MeV}, M_{s}=521 \mathrm{MeV}$ and $\langle\bar{u} u\rangle_{0}=(-247 \mathrm{MeV})^{3}$. Finally we find

$$
G\left(\Lambda_{0}\right)=\frac{1.81}{\Lambda_{0}^{2}}, \quad K\left(\Lambda_{0}\right)=\frac{8.80}{\Lambda_{0}^{5}}
$$

\section{Constituent masses in the LOFF phases}

In this section we evaluate constituent quark masses and gaps for the color superconductive LOFF phase of QCD. At finite chemical potential and in presence of color condensation the lagrangian becomes

$$
\mathcal{L}=\bar{\psi}\left(i \partial_{\mu} \gamma^{\mu}+\hat{\mu} \gamma_{0}\right) \psi+\mathcal{L}_{\text {mass }}+\mathcal{L}_{4}++\mathcal{L}_{6}+\mathcal{L}_{\Delta},
$$

where $\mathcal{L}_{\text {mass }}, \mathcal{L}_{4}$ and $\mathcal{L}_{6}$ have been discussed in Section 2 and we have added a term $\mathcal{L}_{\Delta}$ whose presence is responsible for color condensation. The matrix $\hat{\mu}$ is a matrix diagonal 
both in flavor and color. It depends on $\mu$ (the average quark chemical potential), $\mu_{e}$ (the electron chemical potential), and $\mu_{3}, \mu_{8}$ (color chemical potentials) 㘬. For color and electric neutrality to be implemented it is sufficient to consider only these chemical potentials, related as they are to the charge matrix and the diagonal color operators $T_{3}=$ $\frac{1}{2} \operatorname{diag}(1,-1,0)$ and $T_{8}=\frac{1}{2 \sqrt{3}} \operatorname{diag}(1,1,-2)$ (in general one should introduce a color chemical potential for each $S U(3)$ color charge; however, as shown in [31], for the condensate with the color-flavor structure considered in this paper it is enough to consider only $\mu_{3}$ and $\mu_{8}$, since the charges related to the other color generators automatically vanish). Therefore the matrix $\hat{\mu}$ is written as follows

$$
\hat{\mu}_{i j}^{\alpha \beta}=\left(\mu \delta_{i j}-\mu_{e} Q_{i j}\right) \delta^{\alpha \beta}+\delta_{i j}\left(\mu_{3} T_{3}^{\alpha \beta}+\frac{2}{\sqrt{3}} \mu_{8} T_{8}^{\alpha \beta}\right)
$$

with $Q=\operatorname{diag}(2 / 3,-1 / 3,-1 / 3) \quad(i, j=1,3$ flavor indices; $\alpha, \beta=1,3$ colour indices $)$.

The interaction term in the quark-quark channel $\mathcal{L}_{\Delta}$ is

$$
\mathcal{L}_{\Delta}=\frac{3 G}{4} \sum_{I=1}^{3}\left(\psi_{\alpha i}^{\dagger} i \gamma_{5} \epsilon^{\alpha \beta I} \epsilon_{i j I} C \psi_{\beta j}^{*}\right)\left(\psi_{\gamma m}^{T} C i \gamma_{5} \epsilon^{\gamma \delta I} \epsilon_{m n I} \psi_{\delta n}\right)
$$

Both $\mathcal{L}_{\Delta}$ and $\mathcal{L}_{4}$ in Eq. (2.3) can be obtained by a Fierz rearrangement of the four fermion interaction with the quantum numbers of a single gluon exchange (see for example [27]). This procedure fixes the ratio of the coupling constants, in the quark-antiquark and in the quark-quark channel, to be equal to $3 / 4$. In principle an additional contribution to $\mathcal{L}_{\Delta}$ arises from the six-fermion interaction term, when treated in the mean field approximation. Moreover, there exists also a repulsive interaction in the symmetric color channel. In this paper we neglect both of these terms for simplicity: the effects of these interactions are expected to be small in the high density regime, to which we are interested here.

In the mean field approximation one gets

$$
\mathcal{L}_{\Delta}=-\frac{1}{2} \sum_{I=1}^{3}\left(\Delta_{I}(\boldsymbol{r}) \psi_{\alpha i}^{\dagger} \gamma_{5} \epsilon^{\alpha \beta I} \epsilon_{i j I} C \psi_{\beta j}^{*}+\text { h.c. }\right)+\frac{\Delta_{I}(\boldsymbol{r}) \Delta_{I}^{*}(\boldsymbol{r})}{3 G} .
$$

In order to study the LOFF phase in a generic case we assume

$$
\Delta_{I}(\boldsymbol{r})=\Delta_{I} \sum_{m=1}^{P_{I}} \exp \left(2 i \boldsymbol{q}_{m}^{I} \cdot \boldsymbol{r}\right)
$$

and $P_{I}$ is the number of plane waves for each diquark condensation channel and $2 \boldsymbol{q}_{m}^{I}$ is the quark pair momentum. In the sequel we consider only the case $\boldsymbol{q}_{m}^{I}=q_{I} \hat{\boldsymbol{n}}_{m}^{I}$. Clearly $\Delta_{1}, \Delta_{2}, \Delta_{3}$ refer to $d s, u s, u d$ pairings respectively.

Once the lagrangian is fixed we compute the quark contribution to the free energy in the Hartree approximation:

$$
\Omega=\Omega_{e}+\Omega_{n}+\Omega_{\Delta}
$$


where $\Omega_{e}$ is the free energy of the ideal gas of electrons: $\Omega_{e}=-\mu_{e}^{4} / 12 \pi^{2}$, and $\Omega_{n}$ is the contribution of the unpaired phase:

$$
\Omega_{n}=-4 K \sigma_{u} \sigma_{d} \sigma_{s}+2 G \sum_{f=u, d, s} \sigma_{f}^{2}-2 N_{c} \sum_{f=u, d, s} \int \frac{d^{3} p}{(2 \pi)^{3}}\left\{E_{f}-\left[E_{f}-\mu_{f}\right] \Theta\left(\mu_{f}-E_{f}\right)\right\}
$$

where $E_{f}=\sqrt{p^{2}+M_{f}^{2}}$. The constituent masses $M_{f}$ are here expressed in terms of the $\sigma_{f}$ by means of (2.8). We note that the t'Hooft term gives rise to contributions that mix $q q$ and $\bar{q} q$ condensates. Here, as in [22], we neglect such contributions for simplicity, since they are not expected to change qualitatively the structure of the phase diagram. $\Omega_{\Delta}$ is the contribution from the condensation in the diquark channel. We write this last term in the Ginzburg Landau approximation, as this is the scheme where the LOFF state of QCD with three flavors has been studied so far, see [9], [11] and, for a test of the results of [9], also [10]:

$$
\begin{aligned}
\Omega_{\Delta}= & \frac{2 \mu^{2}}{\pi^{2}}\left[\sum_{I} P_{I} \alpha_{I} \Delta_{I}^{*} \Delta_{I}+\frac{1}{2}\left(\sum_{I} \beta_{I}\left(\Delta_{I}^{*} \Delta_{I}\right)^{2}+\sum_{I>J} \beta_{I J} \Delta_{I}^{*} \Delta_{I} \Delta_{J}^{*} \Delta_{J}\right)\right. \\
& \left.+\frac{1}{3}\left(\sum_{I} \gamma_{I}\left(\Delta_{I}^{*} \Delta_{I}\right)^{3}+\sum_{I \neq J} \gamma_{I J J} \Delta_{I}^{*} \Delta_{I} \Delta_{J}^{*} \Delta_{J} \Delta_{J}^{*} \Delta_{J}+\gamma_{123} \Delta_{1}^{*} \Delta_{1} \Delta_{2}^{*} \Delta_{2} \Delta_{3}^{*} \Delta_{3}\right)\right]
\end{aligned}
$$

We include terms up to sixth order in the gap paramenters, consistently with the results of [1].

It is useful to note that, since we are interested in the three flavor paired quark matter, the chemical potential of the strange quark has to be larger than its constituent mass, otherwise no Fermi sphere for $s$ quarks would exist. In order to determine the ground state we have to minimize $\Omega$ with respect to $\sigma_{f}, \Delta_{I}$ and $q_{I}$ under the conditions of electrical and color neutrality. In the region of interest we expect $\mu_{e} \approx M_{s}^{2} / 4 \mu$ and $\mu_{3} \approx \mu_{8} \approx 0$, i.e. the results for the normal non-superconductive phase. Therefore we assume from the very beginning $\Delta_{1}=0, q_{2}=q_{3} \approx 0.6\left(\mu_{d}-\mu_{u}\right)$ and $\Delta_{2}=\Delta_{3} \equiv \Delta$ [9, 11]. Moreover we are interested to structures with $P_{2}=P_{3} \equiv P$. With these approximations one can write [11]

$$
\Omega_{\Delta}=\frac{2 \mu^{2}}{\pi^{2}}\left[2 P \alpha(\delta \mu) \Delta^{2}+\frac{\bar{\beta}_{e f f}}{2 \delta \mu^{2}} \Delta^{4}+\frac{\bar{\gamma}_{e f f}}{3 \delta \mu^{4}} \Delta^{6}\right]
$$

where $\bar{\beta}_{e f f}$ and $\bar{\gamma}_{e f f}$ are dimensionless coefficients that do not depend on $\delta \mu=M_{s}^{2} / 8 \mu$ and are evaluated in [11] for several crystal structures. In the following we refer to the values reported in Table II of [11]. We notice that the parameters $\bar{\beta}_{\text {eff }}, \bar{\gamma}_{\text {eff }}$ do not depend on the ultraviolet cutoff. Furthermore, the cutoff dependence of the quadratic coefficient in $\Omega_{\Delta}$ is replaced by the dependence on the BCS gap parameter, which we calculate for each value of $\mu$; at the minimum one has

$$
\alpha(\delta \mu)=-\frac{1}{2} \log \left(\frac{\Delta_{2 S C}^{2}}{4 \delta \mu^{2}\left(\eta^{2}-1\right)}\right)
$$


in the above equation $\eta=1.1997$ and $\Delta_{2 S C}$ denotes the gap parameter in the 2SC phase; it is related to the gap of the CFL phase with massless flavors $\Delta_{0}$ by the relation

$$
\Delta_{2 S C}=2^{1 / 3} \Delta_{0} .
$$

Keeping in mind these approximations we are left with the four equations $(f=u, d, s)$

$$
\frac{\partial \Omega}{\partial \sigma_{f}}=0, \quad \frac{\partial \Omega}{\partial \Delta}=0,
$$

which have to be solved simultaneously. Eqs. (3.12) are equivalent to the SchwingerDyson equations for the proper self-energy of the quarks; in particular for $\Delta=0$ one gets the equations for the constituent quark masses in the normal Fermi liquid phase, at finite chemical potential 32]. We note that we have adapted the numerical values of the parameters of the Ginzburg Landau expansion to the numerical values for $\mu$ used in the present paper.

Numerically we find that $\Omega_{\Delta} / \Omega_{n} \approx 10^{-4}$ for the values of $\mu$ of interest. As a result the constituent quark masses in the LOFF phases do not differ significantly from the ones in the unpaired phase $\left(\Omega_{\Delta}\right.$ gives a shift in the masses at most of the 2 percent from the normal phase result). The results are shown in Fig. 3, where we plot the constituent quark masses versus the chemical potential $\mu$. Note that in this figure we use for the ultraviolet cutoff the value (2.17). One can note a first order phase transition at $\mu \approx 350$ characterized by a discontinuity in the values of the constituent light quark masses, and a second order transition at $\mu \approx 500 \mathrm{MeV}$ (at $\mu \approx 350 \mathrm{MeV}$ there is a discontinuity of the strange quark mass too: it is related to the t'Hooft interaction term which links the strange quark mass to the light quarks condensates, see Eq. (2.8)). As discussed in [25], the order of the phase transitions at $T=0$ and large $\mu$ depends in NJL models with normal Fermi liquid behavior on the approximations and the choice of the parameters. This holds also in our case. The difference between $M_{u}$ and $M_{d}$ arises from the finite value of $\mu_{e}$ for $\mu>350$ $\mathrm{MeV}$. We note that in the range of $\mu$ of interest for the LOFF phase $(\mu \sim 500 \mathrm{MeV})$ the condensates $<\bar{u} u>_{0}$ and $<\bar{d} d>_{0}$ vanish, which implies that the constituent masses $M_{u}$ and $M_{d}$ are negligible in comparison with to $M_{s}$. As a consequence the values of the gap parameters depend only on the strange quark mass. As stressed in the introduction, differently from the previous analyses [9, 10, 11] here the strange quark mass is not treated as a free parameter, but determined self-consistently.

Using these results we are able to compute the interval $\left(\mu_{1}, \mu_{2}\right)$ of values of $\mu$ where the LOFF phase is favored. To begin with we examine the simple case of one plane wave per condensate (denoted as $2 \mathrm{PW}$ in [11]) corresponding to $P_{2}=P_{3}=1$ in Eq. (3.5). In Ref. [9, 11, 10] it was found that this LOFF state is energetically favored for the following values of the ratio $M_{s}^{2} / \mu$,

$$
4.80 \Delta_{0} \leq \frac{M_{s}^{2}}{\mu} \leq 7.56 \Delta_{0}
$$

where $\Delta_{0}$ is the CFL gap in the chiral limit. It is given by

$$
\Delta_{0}=2^{2 / 3}\left(\Lambda_{0}-\mu\right) \exp \left(-\frac{\pi^{2}}{6 G\left(\Lambda_{0}\right) \mu^{2}}\right)
$$




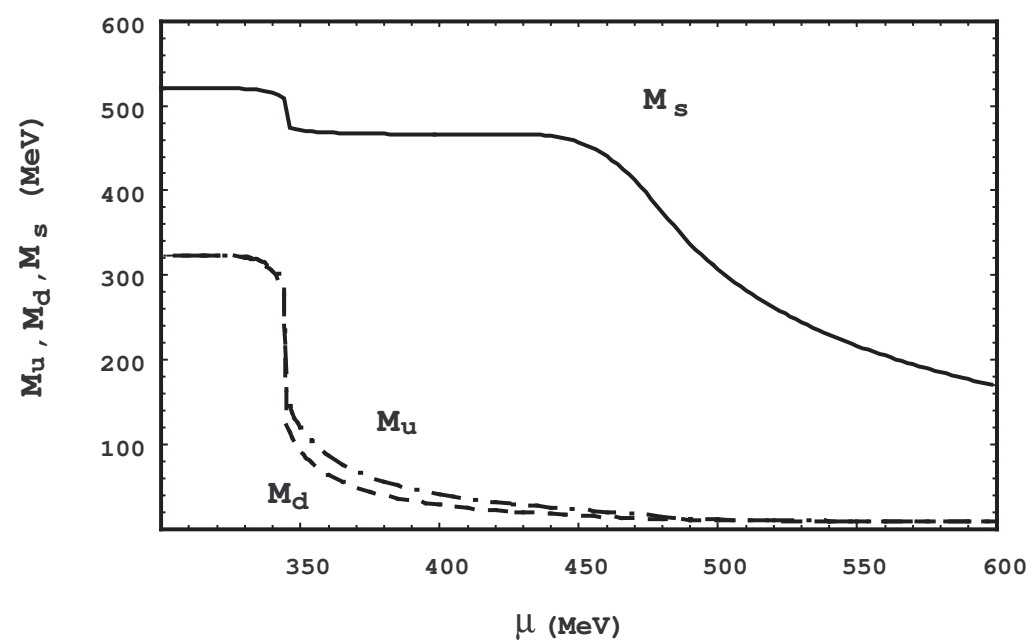

Figure 3: Constituent quark masses $M_{s}, M_{d}$ and $M_{u}$ as functions of the baryon chemical potential $\mu$.

with $\Lambda_{0}$ and $G\left(\Lambda_{0}\right)$ given in (2.17) and 2.18). We note that at the reference value of $\mu=500 \mathrm{MeV}$ we find $\Delta_{0} \approx 50 \mathrm{MeV}$.

The LOFF window is defined by the two points where the horizontal dotted lines cross the vertical axis in Fig. A. The curve represents $M_{s}^{2} /\left(\mu \Delta_{0}\right)$ as a function of $\mu$, with $M_{s}$ and $\Delta_{0}$ both calculated self-consistently as functions of the average quark chemical potential $\mu$. The dotted horizontal lines intersect the curve at the values $\mu_{1}, \mu_{2}$ depicted in the figure. In this way we obtain that the LOFF phase is favored in comparison with the normal phase (this comparison fixes $\mu_{1}$ ) and the gCFL phase (this latter comparison fixes $\mu_{2}$ ) for $\mu \in\left(\mu_{1}, \mu_{2}\right)$, i.e.

$$
467 \mathrm{MeV}<\mu<488 \mathrm{MeV} \text {. }
$$

Therefore there exists a small but finite window in $\mu$ where the LOFF phase is favored in comparison with the normal state and the unstable gCFL phase.

As stressed in the introduction, the analysis of [11] shows that more complicated crystalline structures can lead to a free energy smaller than the $2 \mathrm{PW}$ case. In [11] it was found by a Ginzburg Landau expansion that a crystalline color superconductive phase exists in the following interval:

$$
2.88 \Delta_{0} \leq \frac{M_{s}^{2}}{\mu} \leq 10.36 \Delta_{0}
$$

In more detail, for $2.88 \Delta_{0} \leq M_{s}^{2} / \mu \leq 6.20 \Delta_{0}$ the ground state of three flavor quark matter is the CubeX. In this structure $P_{2}=P_{3}=4$; for each pairing channel the wave vectors $\left\{\boldsymbol{q}_{I}\right\}$ form a square, and the two squares are arranged in such a way that they point to the vertices of a cube. In the remaining region the favored structure is the $2 \mathrm{Cube} 45 \mathrm{z}$ in which 


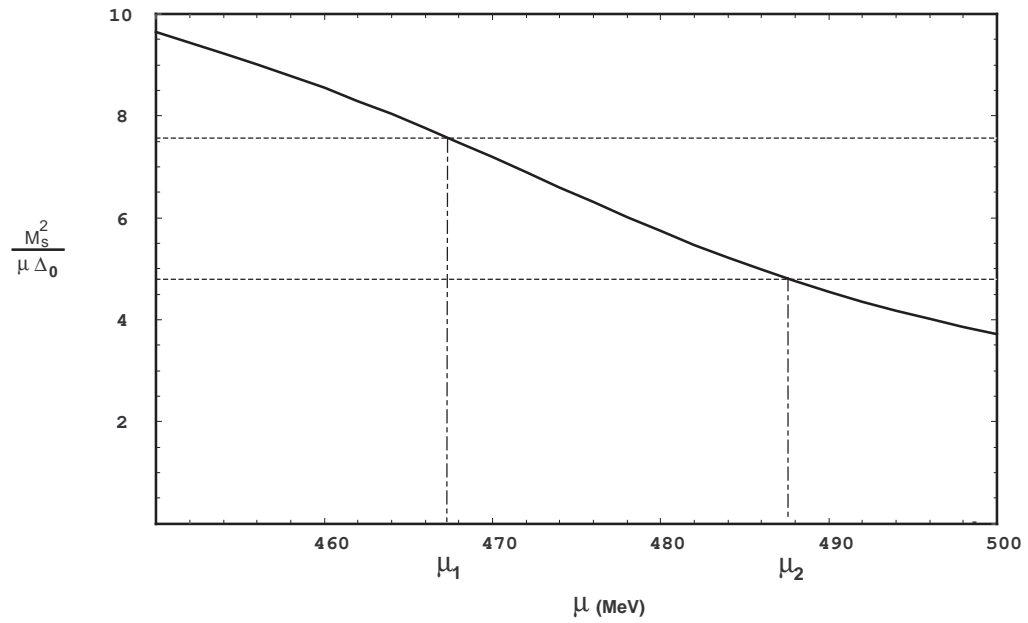

Figure 4: Solid line: $\frac{M_{s}^{2}}{\mu \Delta_{0}}$ versus the baryon chemical potential $\mu(\mathrm{MeV})$ for the LOFF phase with one-plane-wave structure. The UV cutoff is given by Eq. (2.17). The points $\mu_{1}, \mu_{2}$ define the range of $\mu$ where the LOFF phase prevails. For $\mu<\mu_{1}$ the favored phase is the normal one. The upper limit is $\mu_{2}$ is obtained comparing the free energies of the gCFL and the LOFF phase (see text).

$P_{2}=P_{3}=8$; each wave vector set $\left\{\boldsymbol{q}_{I}\right\}$ forms a cube, and the two cubes are rotated by 45 degrees around an axes perpendicular to one of the faces of the cube. Using Eq. (3.16) and the results for the constituent strange quark mass, plotted in Fig. 3, we obtain the LOFF phase is favored for the values of $\mu$ in the window $\left(\mu_{1}, \mu_{2}\right)$ defined by

$$
442 \mathrm{MeV}<\mu<515 \mathrm{MeV} \text {. }
$$

We conclude that there exists a window in $\mu$, larger than that of the $2 \mathrm{PW}$ structure, where the cubic LOFF phase is energetically favored in comparison with the normal state, the 2PW LOFF phase, and the unstable gCFL phase.

The results obtained here for the constituent quark masses are in agreement with those obtained in Ref. [22]. As already stressed, this is due to the fact that the contribution of the quark-quark condensation to the free energy is negligible, and the constituent quark masses are almost equals to their value in the phase with $\Delta=0$.

Let us now discuss the dependence of these results on the cutoff. Thus far we have used an ultraviolet cutoff independent of the quark chemical potential and numerically equal to the value of the model at $\mu=0$ given by eq. (2.17). For large values of $\mu$ (ie $\mu \gg 500 \mathrm{MeV}$ ) this choice leads to a non-monotonic behavior of $\Delta$ with $\mu$. Therefore in [28] a different procedure was suggested. It is based on the following choice: $\Lambda(\mu)=\mu+\delta$, with $\delta=c \mu$. The constant $c$ was fixed imposing that the values of the gap are almost independent of $c$. In this way it was found

$$
c=0.35 \pm 0.10
$$

which is what we assume here, though other choices are possible [33]. Together with this choice of the cutoff at $\mu \neq 0$ one has to use values for the couplings $G$ and $K$ computed at 


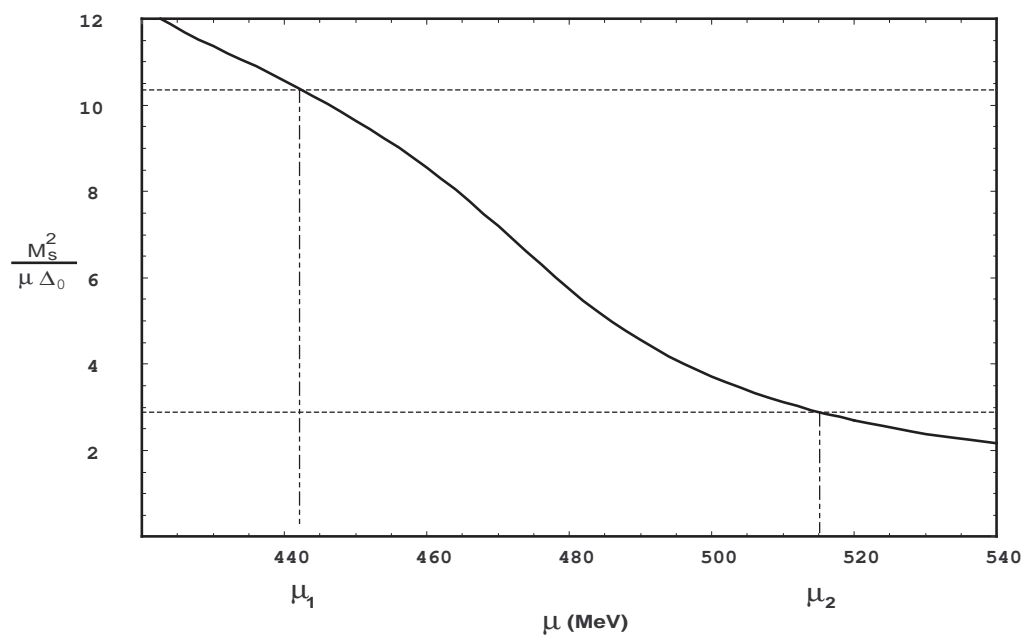

Figure 5: Solid line: ratio $\frac{M_{s}^{2}}{\mu \Delta_{0}}$ versus the baryon chemical potential $\mu(\mathrm{MeV})$ for the LOFF phase with cubic crystalline structure. The UV cutoff, $\mu_{1}, \mu_{2}$ as in Fig. 4.

the same value of $\Lambda$ (the dependence can be read from fig. 2). To test the robustness of our computation we have therefore implemented this second choice of the cutoff. The results we obtain are as follows. The values of the masses are almost identical to the ones found with the cutoff $\Lambda_{0}$, see Fig. 3. As to the windows where the LOFF phase prevails, for $c=0.35$ and the case of the one-plane-wave structure we find $468 \mathrm{MeV}<\mu<481 \mathrm{MeV}$ instead of (3.15); for the case of the cubic structure we find $445 \mathrm{MeV}<\mu<640 \mathrm{MeV}$ instead of the results (3.17). Varying $c$ in the window (3.18) we obtain similar results. Though numerically different from the results obtained with the cutoff $\Lambda_{0}$, the results found with the alternative cutoff procedure do not alter the overall conclusion, ie that there exists a window of values of $\mu$ where the LOFF phase is energetically favored. For the case of the single plane wave the range is between $\approx 470$ and $480 \mathrm{MeV}$. For the case of the cubic structure the window is larger, with a lower limit at $\approx 445 \mathrm{MeV}$ and an upper limit more model dependent, but, in any case not smaller that $545 \mathrm{MeV}$.

Let us finally note that the crystalline color superconductive phase does not have a free energy smaller than gCFL for all the values of the strange quark mass. If the gCFL phase has smaller free energy, as it is unstable, it must be replaced by a new state, most likely to be a current-carrying meson condensate state and/or gluonic phases [34].

\section{Conclusions}

We have evaluated the three flavor QCD LOFF phase by a self-consistent computation of the quark masses. We have adopted two different methods to cutoff the theory in the ultraviolet regime, with results that are basically consistent. We have determined the LOFF window as a function of the baryon chemical potential only, instead of the usual $M_{s}^{2} / \mu$ representation where the constituent quark masses are taken as external parameters. We 
find that there exist ranges of values of the quark chemical potential where the crystalline color superconductive phase has a free energy smaller than the homogeneous and the normal phases.. We find the range of $\mu$ given by Eq. (3.15) for the one plane wave structure of the LOFF condensate, with the the cutoff (2.17), and a slightly smaller range for the alternate cutoff $\Lambda=\mu+c \mu$ with $c$ in (3.18). As shown in [11] the cubic structure is energetically favored in comparison to the single plane wave. The numerical results of [11] might be called into question since the phase transition to the normal phase for the cubic structures is first order and therefore the use of the Ginzburg Landau approximation is questionable in this case. In any event also for the cubic structure there exists a range of values of the quark chemical potential, expressed by Eq. (3.17) and, as expected, larger than the range (3.15), where the cubic crystalline LOFF structure is energetically favored (for the alternate cutoff the range is even larger). Let us finally note that, as shown by Fig. 3, in the region $350 \mathrm{MeV}<\mu<445 \mathrm{MeV}$ the Fermi surfaces of the $u$ and $d$ quarks are present, whereas the one of the strange quark is not. Even though the LOFF state with three flavors is not possible in this case, still there exists the possibility of a LOFF state with a $\langle u d\rangle$ color condensate. This case has been well studied in the literature [8]. We have computed in this case the difference in chemical potential between the $u$ and $d$ quarks: $\delta \mu=\mu_{e} / 2$, and we have noted that in correspondence of these values one or more crystalline structures of the LOFF state with two flavors (and more than one plane wave) are possible. Therefore the window for $\mu$ available for the LOFF color superconductivity is actually larger than shown by figures 4 and 5 .

Our results could be relevant for astrophysical applications of the LOFF phase, because the ranges of $\mu$ we have found approximately coincide with those considered for the core of compact stellar objects. If the density in some region of a compact star do correspond to baryon chemical potential belonging to the range (3.17), one should conclude the quark matter is in an inhomogeneous color superconductive state with a cubic crystal structure. For a better understanding of the role of the QCD LOFF phase in compact stars it is however of vital importance to compute the transport properties of the crystalline three flavor color superconductor. Since in the LOFF phase both the rotational and the translational symmetries are broken by the condensate, phonons appear in the spectrum. Recently the effective lagrangian of such phonons has been evaluated in [35]. It has been found that the crystalline color superconductor responds to shear stress as a very rigid solid because of its high shear modulus; the results of [35] show that some pulsar glitches may originate within a quark matter core deep within a neutron star. Beside the phonons, in the LOFF phase there exist an octet of pseudo-Goldstone bosons related to the breaking of $S U(3)_{A}$ : it would be interesting to investigate if a kaon condensation occurs in the ground state (in the CFL phase this problem has been extensively studied, see for example [36]). We will come back to this problem in the future. Besides the transport coefficients, it might be interesting the evaluation of the cooling curves of a compact star whose core is in a cubic LOFF state (the simple case of two plane waves has been studied in [37]). Another interesting investigation is the use of different density dependent cutoffs as in Ref. [33]. We leave also this study to future work. 


\section{Acknowledgments}

We thank R. Anglani, D. Blaschke, M. Buballa, R. Casalbuoni, M. Ciminale, R. Gatto, V. Laporta, M. Mannarelli, K. Rajagopal and R. Sharma for enlightening discussions and comments.

\section{References}

[1] J. C. Collins and M. J. Perry, Phys. Rev. Lett. 34, 1353 (1975); B. C. Barrois, Nucl. Phys. B 129, 390 (1977); D. Bailin and A. Love, Phys. Rept. 107, 325 (1984); M. G. Alford, K. Rajagopal and F. Wilczek, Phys. Lett. B 422, 247 (1998) [arXiv:hep-ph/9711395]; R. Rapp, T. Schafer, E. V. Shuryak and M. Velkovsky, Phys. Rev. Lett. 81, 53 (1998) [arXiv:hep-ph/9711396]. D. T. Son, Phys. Rev. D 59, 094019 (1999) [arXiv:hep-ph/9812287]; R. D. Pisarski and D. H. Rischke, Phys. Rev. D 61, 074017 (2000) [arXiv:nucl-th/9910056].

[2] K. Rajagopal and F. Wilczek, arXiv:hep-ph/0011333; M. G. Alford, Ann. Rev. Nucl. Part. Sci. 51, 131 (2001) [arXiv:hep-ph/0102047]; G. Nardulli, Riv. Nuovo Cim. 25N3, 1 (2002) [arXiv:hep-ph/0202037]; T. Schafer, arXiv:hep-ph/0509068.

[3] M. G. Alford, K. Rajagopal and F. Wilczek, Nucl. Phys. B 537, 443 (1999) [arXiv:hep-ph/9804403].

[4] M. Alford, C. Kouvaris and K. Rajagopal, Phys. Rev. Lett. 92, 222001 (2004) [arXiv:hep-ph/0311286]; M. Alford, C. Kouvaris and K. Rajagopal, Phys. Rev. D 71, 054009 (2005) [arXiv:hep-ph/0406137]; K. Fukushima, C. Kouvaris and K. Rajagopal, Phys. Rev. D 71, 034002 (2005) [arXiv:hep-ph/0408322].

[5] I. Shovkovy and M. Huang, Phys. Lett. B 564, 205 (2003) [arXiv:hep-ph/0302142]; M. Huang and I. Shovkovy, Nucl. Phys. A 729, 835 (2003) [arXiv:hep-ph/0307273].

[6] T. Schafer, Phys. Rev. D 62, 094007 (2000) [arXiv:hep-ph/0006034]; M. G. Alford, J. A. Bowers, J. M. Cheyne and G. A. Cowan, Phys. Rev. D 67, 054018 (2003) [arXiv:hep-ph/0210106]; A. Schmitt, Phys. Rev. D 71, 054016 (2005) [arXiv:nucl-th/0412033]; D. N. Aguilera, D. Blaschke, M. Buballa and V. L. Yudichev, Phys. Rev. D 72, 034008 (2005) [arXiv:hep-ph/0503288]; F. Marhauser, D. Nickel, M. Buballa and J. Wambach, arXiv:hep-ph/0612027.

[7] A. I. Larkin and Yu. N. Ovchinnikov, Zh. Eksp. Teor. Fiz. 47, 1136 (1964) (Sov. Phys. JETP 20, 762 (1965)); P.Fulde and R. A. Ferrell, Phys. Rev. 135, A550 (1964).

[8] M. G. Alford, J. A. Bowers and K. Rajagopal, Phys. Rev. D 63, 074016 (2001) [arXiv:hep-ph/0008208]; J. A. Bowers, J. Kundu, K. Rajagopal and E. Shuster, Phys. Rev. D 64, 014024 (2001) [arXiv:hep-ph/0101067]; A. K. Leibovich, K. Rajagopal and E. Shuster, Phys. Rev. D 64, 094005 (2001) [arXiv:hep-ph/0104073]; R. Casalbuoni, R. Gatto, M. Mannarelli and G. Nardulli, Phys. Rev. D 66, 014006 (2002) [arXiv:hep-ph/0201059]; J. A. Bowers and K. Rajagopal, Phys. Rev. D 66, 065002 (2002) [arXiv:hep-ph/0204079]; R. Casalbuoni, M. Ciminale, M. Mannarelli, G. Nardulli, M. Ruggieri and R. Gatto, Phys. Rev. D 70, 054004 (2004) [arXiv:hep-ph/0404090].

[9] R. Casalbuoni, R. Gatto, N. Ippolito, G. Nardulli and M. Ruggieri, Phys. Lett. B 627, 89 (2005) [Erratum-ibid. B 634, 565 (2006)] [arXiv:hep-ph/0507247].

[10] M. Mannarelli, K. Rajagopal and R. Sharma, Phys. Rev. D 73, 114012 (2006) [arXiv:hep-ph/0603076]. 
[11] K. Rajagopal and R. Sharma, Phys. Rev. D 74, 094019 (2006) [arXiv:hep-ph/0605316].

[12] R. Casalbuoni, M. Ciminale, R. Gatto, G. Nardulli and M. Ruggieri, Phys. Lett. B 642, 350 (2006) [arXiv:hep-ph/0606242].

[13] I. Giannakis and H. C. Ren, Phys. Lett. B 611, 137 (2005) [arXiv:hep-ph/0412015].

[14] I. Giannakis and H. C. Ren, Nucl. Phys. B 723, 255 (2005) [arXiv:hep-th/0504053].

[15] I. Giannakis, D. f. Hou and H. C. Ren, Phys. Lett. B 631, 16 (2005) [arXiv:hep-ph/0507306].

[16] E. V. Gorbar, M. Hashimoto and V. A. Miransky, Phys. Rev. Lett. 96, 022005 (2006) [arXiv:hep-ph/0509334].

[17] M. Ciminale, G. Nardulli, M. Ruggieri and R. Gatto, Phys. Lett. B 636, 317 (2006) [arXiv:hep-ph/0602180].

[18] M. Huang and I. A. Shovkovy, Phys. Rev. D 70, 051501 (2004) [arXiv:hep-ph/0407049]; R. Casalbuoni, R. Gatto, M. Mannarelli, G. Nardulli and M. Ruggieri, Phys. Lett. B 605, 362 (2005) [Erratum-ibid. B 615, 297 (2005)] [arXiv:hep-ph/0410401]; K. Fukushima, Phys. Rev. D 72, 074002 (2005) [arXiv:hep-ph/0506080]; M. Alford and Q. h. Wang, J. Phys. G 31, 719 (2005) [arXiv:hep-ph/0501078].

[19] A. W. Steiner, S. Reddy and M. Prakash, Phys. Rev. D 66, 094007 (2002) [arXiv:hep-ph/0205201].

[20] M. Buballa and M. Oertel, Nucl. Phys. A 703, 770 (2002) [arXiv:hep-ph/0109095].

[21] H. Abuki, M. Kitazawa and T. Kunihiro, Phys. Lett. B 615, 102 (2005) [arXiv:hep-ph/0412382].

[22] S. B. Ruster, V. Werth, M. Buballa, I. A. Shovkovy and D. H. Rischke, Phys. Rev. D 72 (2005) 034004 [arXiv:hep-ph/0503184].

[23] D. Blaschke, S. Fredriksson, H. Grigorian, A. M. Oztas and F. Sandin, Phys. Rev. D 72, 065020 (2005) [arXiv:hep-ph/0503194].

[24] Y. Nambu and G. Jona-Lasinio, Phys. Rev. 122, 345 (1961); Y. Nambu and G. Jona-Lasinio, Phys. Rev. 124, 246 (1961).

[25] S. P. Klevansky, Rev. Mod. Phys. 64 (1992) 649.

[26] T. Hatsuda and T. Kunihiro, Phys. Rept. 247, 221 (1994) [arXiv:hep-ph/9401310].

[27] M. Buballa, Phys. Rept. 407, 205 (2005) [arXiv:hep-ph/0402234].

[28] R. Casalbuoni, R. Gatto, G. Nardulli and M. Ruggieri, Phys. Rev. D 68 (2003) 034024 [arXiv:hep-ph/0302077].

[29] P. Colangelo, F. De Fazio, G. Nardulli and N. Paver, Phys. Lett. B 408, 340 (1997) [arXiv:hep-ph/9704249].

[30] W. M. Yao et al. [Particle Data Group], J. Phys. G 33, 1 (2006).

[31] M. Buballa and I. A. Shovkovy, Phys. Rev. D 72, 097501 (2005) [arXiv:hep-ph/0508197].

[32] M. Asakawa and K. Yazaki, Nucl. Phys. A 504, 668 (1989); S. Klimt, M. Lutz and W. Weise, Phys. Lett. B 249, 386 (1990).

[33] M. Baldo, G. F. Burgio, P. Castorina, S. Plumari and D. Zappala, arXiv:hep-ph/0607343. 
[34] M. Huang, Phys. Rev. D 73, 045007 (2006) [arXiv:hep-ph/0504235]; D. K. Hong, arXiv:hep-ph/0506097; M. Alford and Q. h. Wang, J. Phys. G 32, 63 (2006) [arXiv:hep-ph/0507269]; A. Kryjevski, arXiv:hep-ph/0508180; T. Schafer, Phys. Rev. Lett. 96, 012305 (2006) [arXiv:hep-ph/0508190]; E. V. Gorbar, M. Hashimoto and V. A. Miransky, Phys. Lett. B 632, 305 (2006) [arXiv:hep-ph/0507303]; A. Gerhold, T. Schaefer and A. Kryjevski, arXiv:hep-ph/0612181.

[35] M. Mannarelli, K. Rajagopal and R. Sharma, arXiv:hep-ph/0702021.

[36] P. F. Bedaque and T. Schafer, Nucl. Phys. A 697, 802 (2002) [arXiv:hep-ph/0105150].

[37] R. Anglani, G. Nardulli, M. Ruggieri and M. Mannarelli, Phys. Rev. D 74, 074005 (2006) [arXiv:hep-ph/0607341]. 